\section{TEP crônica hipertensiva}

Define-se como TEP crônica hipertensiva, ou mais frequentemente, HPTC, o quadro de hipertensão pulmonar observado após um período mínimo de 3 meses após pelo menos um episódio de embolia de pulmão, desde que excluídas outras causas de hipertensão pulmonar. ${ }^{(1)} \mathrm{A}$ resolução parcial do defeito perfusional é comum, podendo chegar a 50\% após 1 ano de seguimento. ${ }^{(2)}$ Observa-se que 3,8\% dos pacientes que sobrevivem a um episódio agudo de embolia pulmonar evoluem, após 2 anos de seguimento, para um quadro crônico hipertensivo. ${ }^{(3,4)}$ Sendo assim, conclui-se que a maior parte dos pacientes com déficits perfusionais persistentes não desenvolve HPTC, devendo, portanto, haver outros fatores associados, hereditários ou adquiridos, para sua ocorrência. Por outro lado, até $40 \%$ dos pacientes com HPTC não tem história previa de TEV. ${ }^{(5)}$

Na HPTC, o principal sintoma é a dispneia progressiva, podendo estar acompanhada de tosse seca. A síncope e a opressão retroesternal aos esforços são mais comuns nos casos terminais, nos quais se observa a pressão na artéria pulmonar em valores muito elevados. Esses sinais e sintomas encontrados são muito inespecíficos, e isoladamente, revelam pouco valor na identificação de HPTC, pois são comuns às diversas etiologias que compõem o quadro de hipertensão pulmonar. Entretanto, em indivíduos com fatores predisponentes bem caracterizados, história de TEP prévia e associados a exames de imagem torácicos, esses achados clínicos, apesar de inespecíficos, podem trazer uma importante contribuição para a elucidação diagnóstica de HPTC. ${ }^{(6)}$

0 ecocardiograma é o principal método diagnóstico não invasivo, que possibilita a análise da função do VD com a estimativa da pressão em artéria pulmonar. ${ }^{(7)}$ Junto com a radiografia de tórax, esse exame faz parte da avaliação inicial de pacientes suspeitos de apresentar hipertensão pulmonar após a avaliação clínica.

A cintilografia pulmonar perfusional é o exame de eleição para a exclusão de HPTC, quando o resultado for normal ou de baixa probabilidade.(B) ${ }^{(8)}$ Pacientes candidatos à cirurgia têm ao menos um defeito segmentar ou maior, associado à normalidade no estudo ventilatório. Deve chamar a atenção para outras etiologias (neoplasia, agenesia, mediastinite) quando houver defeito pulmonar de todo um pulmão somente.

A angio-TC permite observar a presença de trombos, sua localização, a circulação brônquica colateral, alterações parenquimatosas (padrão em mosaico, áreas de infarto), bem como comorbidades (enfisema, fibrose pulmonar). Eventualmente, algumas características permitem a separação do fenômeno tromboembólico agudo de HPTC..$^{(9)}$ Resultados de angio-TC sem a presença de trombos não exclui completamente a possibilidade de HPTC; entretanto, os novos tomógrafos com multidetectores possuem acurácia progressivamente melhor para esse contexto. ${ }^{(10)} \mathrm{A}$ ressonância magnética, tal como a angio-TC, permite a visualização direta da falha de enchimento do vaso, com a vantagem de não se irradiar o paciente, nem de se utilizar contraste iodado. Entretanto, é uma técnica mais dispendiosa, demorada e menos disponível que a TC. A arteriografia pulmonar é o exame padrão para o estadiamento vascular e sempre deve ser realizada quando existe a indicação cirúrgica.(B) Embora invasiva, é segura em mãos experientes, mesmo em pacientes hemodinamicamente instáveis..$^{10,11)}$

A avaliação hemodinâmica invasiva é essencial na avaliação de pacientes com HPTC. (A) Permite a confirmação do diagnóstico de hipertensão pulmonar, determina a gravidade (principalmente o grau de elevação da pressão na artéria pulmonar e no átrio direito, assim como o comprometimento do débito cardíaco) e permite predizer razoavelmente a relação riscobenefício do tratamento cirúrgico (tanto pelos valores absolutos, quanto pela análise do gráfico da curva de oclusão da artéria pulmonar). ${ }^{(10-12)}$

0 tratamento preferencial para a maioria dos casos de HPTC é a tromboendarterectomia, embora, para casos selecionados, o transplante pulmonar possa ser considerado como alternativa. $(\mathrm{A})^{(13,14)} \mathrm{Em}$ pacientes selecionados portadores de HPTC, com obstrução vascular central, recomenda-se como tratamento a tromboendarterectomia, desde que realizada em centros especializados. $(C)^{(10)}$

A anticoagulação oral, mantendo-se a RNI entre 2-3, deve ser prescrita para todos os portadores de HPTC que foram submetidos à tromboendarterectomia, assim como para aqueles cujo procedimento cirúrgico não foi realizado.(B) ${ }^{(10)}$

Apesar de alguns centros ainda colocarem $\mathrm{FVCl}$ profilaticamente em todos os pacientes, essa ainda é uma conduta controversa, já abandonada por alguns grupos mais experientes..$^{(5,11)}$

0 uso de fármacos deve ser prescrito para pacientes que não são candidatos à cirurgia (tanto por inacessibilidade das lesões, como por 
contraindicações por outras comorbidades).(B) As várias drogas utilizadas no tratamento da hipertensão arterial pulmonar mostraram benefícios em pacientes com HPTC, mas com evidência baseada em séries de casos e em estudos de coorte. Nesse contexto, inclui-se a utilização de óxido nítrico, epoprostenol, sildenafil, iloprost, treprostinil e bosentana. ${ }^{(15-22)}$

0 uso de fármacos como "ponte" para a cirurgia ainda é um tema em debate. Em geral, não se deve retardar o procedimento visando otimizar o paciente. Entretanto, alguns pacientes limítrofes podem ser levados à cirurgia em melhores condições. A decisão deverá ser tomada conjuntamente com o cirurgião, levando-se em conta o quanto poderá ser reduzida a resistência vascular pulmonar com o procedimento para que o paciente tolere o período do pós-operatório imediato. ${ }^{(10)}$

Alguns fatores predizem de modo independente um pior prognóstico dos pacientes com HPTC. Os principais fatores são a não realização de tromboendarterectomia, hipertensão pulmonar grave e HPTC associada a condições clínicas (por ex., esplenectomia, doenças inflamatórias e uso de cateteres venosos permanentes). ${ }^{(12)}$

Em 2005, a Sociedade Brasileira de Pneumologia e Tisiologia elaborou uma diretriz específica para o atendimento de pacientes com hipertensão pulmonar, que inclui aqueles com HPTC. (23)

\section{Referências}

1. Terra-Filho M. Uso racional dos métodos diagnósticos na doença tromboembólica pulmonar. ln: Sociedade Brasileira de Pneumologia e Tisiologia. Pneumologia - Atualização e Reciclagem. Rio de Janeiro: Revinter; 2004.

2. Nijkeuter M, Hovens MM, Davidson BL, Huisman MV. Resolution of thromboemboli in patients with acute pulmonary embolism: a systematic review. Chest. 2006;129(1):192-7.

3. Pengo V, Lensing AW, Prins MH, Marchiori A, Davidson BL, Tiozzo F, et al. Incidence of chronic thromboembolic pulmonary hypertension after pulmonary embolism. N Engl J Med. 2004;350(22):2257-64.

4. Becattini C, Agnelli G, Pesavento R, Silingardi M, Poggio $\mathrm{R}$, Taliani MR, et al. Incidence of chronic thromboembolic pulmonary hypertension after a first episode of pulmonary embolism. Chest. 2006;130(1):172-5.

5. Hoeper MM, Mayer E, Simonneau G, Rubin LJ. Chronic thromboembolic pulmonary hypertension. Circulation. 2006;113(16):2011-20.

6. Auger WR, Kim NH, Kerr KM, Test VJ, Fedullo PF. Chronic thromboembolic pulmonary hypertension. Clin Chest Med. 2007;28(1):255-69, x.

7. Sbano JC, Tsutsui JM, Terra-Filho M, Mathias-Junior W. Papel da ecodopplercardiografia na avaliação da hipertensão arterial pulmonar. J Pneumol. 2004;30(1):78-86.
8. Rich S, Rabinovitch M. Diagnosis and treatment of secondary (non-category 1) pulmonary hypertension. Circulation. 2008;118(21):2190-9.

9. Heinrich M, Uder M, Tscholl D, Grgic A, Kramann B, Schäfers HJ. CT scan findings in chronic thromboembolic pulmonary hypertension: predictors of hemodynamic improvement after pulmonary thromboendarterectomy. Chest. 2005;127(5):1606-13.

10. Hoeper MM, Barberà JA, Channick RN, Hassoun PM, Lang 1M, Manes A, et al. Diagnosis, assessment, and treatment of non-pulmonary arterial hypertension pulmonary hypertension. J Am Coll Cardiol. 2009;54(1 Suppl):S85-96.

11. Dartevelle P, Fadel E, Mussot S, Chapelier A, Hervé P, de Perrot $\mathrm{M}$, et al. Chronic thromboembolic pulmonary hypertension. Eur Respir J. 2004;23(4):637-48.

12. Bonderman D, Skoro-Sajer N, Jakowitsch J, Adlbrecht C, Dunkler D, Taghavi S, et al. Predictors of outcome in chronic thromboembolic pulmonary hypertension. Circulation. 2007;115(16):2153-8.

13. Jamieson SW, Kapelanski DP, Sakakibara N, Manecke GR, Thistlethwaite PA, Kerr KM, et al. Pulmonary endarterectomy: experience and lessons learned in 1,500 cases. Ann Thorac Surg. 2003;76(5):1457-62; discussion 1462-4.

14. Genta PR, Jatene FB, Terra-Filho M. Quality of life and shortness of breath before and after pulmonary thromboendarterectomy. Am J Respir Crit Care Med. 2002;165(Suppl 1):A329.

15. Kramm T, Eberle B, Krummenauer F, Guth S, Oelert $\mathrm{H}$, Mayer E. Inhaled iloprost in patients with chronic thromboembolic pulmonary hypertension: effects before and after pulmonary thromboendarterectomy. Ann Thorac Surg. 2003;76(3):711-8.

16. Ghofrani HA, Schermuly RT, Rose F, Wiedemann R, Kohstall MG, Kreckel A, et al. Sildenafil for long-term treatment of nonoperable chronic thromboembolic pulmonary hypertension. Am J Respir Crit Care Med. 2003;167(8):1139-41.

17. Hoeper MM, Kramm T, Wilkens H, Schulze C, Schäfers $\mathrm{HJ}$, Welte T, et al. Bosentan therapy for inoperable chronic thromboembolic pulmonary hypertension. Chest. 2005;128(4):2363-7.

18. Imanaka H, Miyano H, Takeuchi M, Kumon K, Ando M. Effects of nitric oxide inhalation after pulmonary thromboendarterectomy for chronic pulmonary thromboembolism. Chest. 2000;118(1):39-46.

19. Hughes RJ, Jais X, Bonderman D, Suntharalingam J, Humbert M, Lang 1 , et al. The efficacy of bosentan in inoperable chronic thromboembolic pulmonary hypertension: a 1-year follow-up study. Eur Respir J. 2006;28(1):138-43.

20. Skoro-Sajer N, Bonderman D, Wiesbauer F, Harja E, Jakowitsch J, Klepetko W, et al. Treprostinil for severe inoperable chronic thromboembolic pulmonary hypertension. J Thromb Haemost. 2007;5(3):483-9.

21. Krug S, Hammerschmidt S, Pankau H, Wirtz $H$, Seyfarth HJ. Acute improved hemodynamics following inhaled iloprost in chronic thromboembolic pulmonary hypertension. Respiration. 2008;76(2):154-9.

22. Suntharalingam J, Treacy CM, Doughty NJ, Goldsmith K, Soon E, Toshner MR, et al. Long-term use of sildenafil in inoperable chronic thromboembolic pulmonary hypertension. Chest. 2008;134(2):229-36.

23. Sociedade Brasileira de Pneumologia e Tisiologia. Hipertensão pulmonar tromboembólica crônica. J Bras Pneumol. 2005;31(Suppl 2):S28-S31. 